\title{
Analytical Treatment of Randomly Birefringent Periodically Spun Fibers
}

\author{
Anna Pizzinat, Luca Palmieri, Brian S. Marks, Curtis R. Menyuk, Fellow, IEEE, Fellow, OSA, and \\ Andrea Galtarossa, Member, IEEE
}

\begin{abstract}
Spun fibers are increasingly used in telecommunication systems because their polarization-mode dispersion (PMD) is lower than that in unspun fibers. In this paper, we investigate the effects of a periodic spin on the PMD of fibers with randomly varying birefringence. Numerical simulations show that when the spin period is of the same order as or larger than the beat length, the mean differential group delay of a spun fiber depends on the model used for the random birefringence. We then carry out a general theoretical analysis using the second Wai-Menyuk model, which is the only model of fiber birefringence to date that is consistent with polarization optical time domain reflectometry data. Finally, we consider some particular regimes by means of a perturbative approach.
\end{abstract}

Index Terms-Beat length, birefringence correlation length, differential group delay, fiber birefringence, polarization-mode dispersion (PMD), spun fibers.

\section{INTRODUCTION}

W ITH the increase in bit rate and transmission distance of optical systems, polarization-mode dispersion (PMD) is becoming one of the most critical challenges for the deployment of robust networks [1]. In the last few years, a great effort has been spent to analyze and propose new techniques for PMD mitigation. The design and development of PMD compensators for installed systems [2], [3] is a difficult task because of the random nature of the fiber birefringence. A different approach to PMD mitigation is to develop low-PMD fibers, which may be done by spinning a fiber as it is drawn [4]. Some time ago, it was found that it is more effective to spin fibers periodically as they are drawn, rather than spinning them at a constant rate [5], [6]. More recently, it was theoretically proven that optimized periodic spin functions yield a differential group delay that does not increase with distance in fibers with deterministic birefringence [7].

Manuscript received January 10, 2003; revised August 4, 2003. This work was supported in part by the European project Information Society Technology/All optical Terabit per second Lambda Shifted Transmission (IST/ATLAS) and the Minestro Università e Ricerca (MIUR) 40\% project under Project 2001098217-002.

A. Pizzinat is with the Department of Information Engineering, University of Padova, 35131 Padova, Italy, and also with the Department of Computer Science and Electrical Engineering, University of Maryland, Baltimore, MD 21250 USA (e-mail: annap@wave.dei.unipd.it.).

L. Palmieri and A. Galtarossa are with the Department of Information Engineering, University of Padova, 35131 Padova, Italy.

B. S. Marks is with the Department of Computer Science and Electrical Engineering, University of Maryland, Baltimore, MD 21250 USA, and also with the Laboratory for Physical Sciences, College Park, MD 20740 USA.

C. R. Menyuk is with the Department of Computer Science and Electrical Engineering, University of Maryland, Baltimore, MD 21250 USA.

Digital Object Identifier 10.1109/JLT.2003.821723
When one wants to study the effects of a periodic spin, one must take into consideration four quantities: the beat length $L_{B}$, which is inversely proportional to the mean birefringence strength; the birefringence correlation length $L_{F}$, which describes the length scale over which an ensemble of fibers with randomly varying birefringence becomes uncorrelated; the spin period $p$; and the spin amplitude $A_{0}$. In recent papers, spun fibers have been studied in special limits, where the analysis can be simplified. In [7] and [8], the case of a short spin period compared with the beat length was considered $\left(p \ll L_{B}\right)$. In [7], [9], and [10], the fiber birefringence was assumed to be deterministic, corresponding to $L_{F} \rightarrow \infty$. Only in [8] was the random birefringence of real telecommunication fibers considered, and it was modeled with a fixed strength and varying orientation (fixed modulus model, or FMM), in accordance with the first of the two Wai-Menyuk models [11].

However, many experiments have shown that the birefringence strength is not fixed. Instead, it varies at random [12], in agreement with the second Wai-Menyuk model (random modulus model, or RMM) [11]. In addition, in general, it is difficult to predict the beat length $L_{B}$ and the correlation length $L_{F}$ of a fiber. Measurements performed to date show that both $L_{B}$ and $L_{F}$ can vary over a wide range of values, from approximately 1 $\mathrm{m}$ up to tens of meters, depending on the fiber type and on the environmental conditions [13], [14].

As a consequence, there are two topics that should be addressed. First, it is important to characterize the behavior of spun fibers when the short-period assumption is not satisfied and to understand in which regimes the spin is effective in reducing the mean differential group delay (DGD). Second, it is important to study the behavior of randomly birefringent spun fibers predicted by the RMM and to understand the differences between the FMM and the RMM.

The first aim of this paper is to understand whether, and in which regimes, the two models of birefringence lead to the same mean DGD as in the case of unspun fibers. The second aim is to obtain as much information as possible of the consequences of the RMM by performing a theoretical analysis based on this model. After a brief description of the two physical models of birefringence, we perform a set of numerical simulations in order to understand the spin effects as a function of the birefringence $\left(L_{B}\right.$ and $\left.L_{F}\right)$ and the spin parameters $\left(A_{0}\right.$ and $\left.p\right)$ for both models of birefringence. This analysis shows that in some regimes the two models lead to significantly different results.

We analytically study PMD in spun fibers by means of the RMM. We obtain an infinite sequence of coupled equations that can be usefully truncated in some cases but that appear to di- 
verge in other cases. in addition, we apply perturbation techniques and show how the introduction of a periodic spin influences the PMD of a randomly birefringent fiber in the different regimes when one of the length scales, e.g., the birefringence correlation length or the spin period, is much shorter than the others.

\section{Two MODELS FOR THE BIREFRINGENCE}

The evolution along the fiber of the polarization dispersion vector $\Omega(z, \omega)$ is governed by the dynamical equation [15]

$$
\frac{\partial \boldsymbol{\Omega}(z, \omega)}{\partial z}=\frac{\partial \boldsymbol{\beta}(z, \omega)}{\partial \omega}+\boldsymbol{\beta}(z, \omega) \times \boldsymbol{\Omega}(z, \omega)
$$

where $\omega$ is the angular frequency, and $\beta(z, \omega)=\left(\beta_{1}, \beta_{2}, \beta_{3}\right)^{T}$ is the local birefringence vector. For standard telecommunication fibers $\boldsymbol{\beta}(z, \omega)$ is a random function of $z$, whose properties may be described by appropriate statistical models. In this paper, we consider the two Wai-Menyuk models [11], which are described here briefly for completeness.

Both models assume that no circular birefringence is present, i.e., $\boldsymbol{\beta}=\left(\beta_{1}, \beta_{2}, 0\right)^{T}$. This assumption is not restrictive because the circular birefringence in telecommunication fibers is negligible [16]. Both models also assume that only the modulus of birefringence depends on $\omega$, so that we can write $\beta(z, \omega)=$ $b(z, \omega) \hat{\boldsymbol{\beta}}(z)$, where $b(z, \omega)$ is the modulus and $\hat{\boldsymbol{\beta}}(z)$ the direction. We indicate the $\omega$ derivative of $b(z, \omega)$ as $b_{\omega}$, and in our simulations we assume that

$$
b_{\omega}(z)=\frac{\partial b(z, \omega)}{\partial \omega}=\frac{b(z, \omega)}{\omega}
$$

as it is commonly done in the literature [11]. This assumption is not needed for our analysis and does not qualitatively affect the results.

The second Wai-Menyuk model is the RMM, which describes $\beta_{1}(z)$ and $\beta_{2}(z)$ as independent Langevin processes [11]

$$
\frac{d \beta_{i}}{d z}=-\rho \beta_{i}(z)+\sigma \eta_{i}(z), \quad i=1,2
$$

where $\eta_{1}(z)$ and $\eta_{2}(z)$ are independent Gaussian white noise processes. As a consequence, the modulus $b(z, \omega)$ is a Rayleigh distributed random variable. Parameters $\rho$ and $\sigma$ define the statistical properties of the birefringence. In particular, the birefringence correlation length is $L_{F}=1 / \rho$, and the beat length reads $L_{B}=2 \pi /\left\langle b^{2}\right\rangle^{1 / 2}=2 \pi \sqrt{\rho} / \sigma=\sqrt{2} \pi / \sigma_{\beta}$. Please note that the new parameter $\sigma_{\beta}$ is related to the statistical properties of the modulus of birefringence by means of $\left\langle b^{2 h}\right\rangle=h !\left(2 \sigma_{\beta}^{2 h}\right)$. Furthermore, in the long-length regime, the mean DGD becomes [11]

$$
\langle\Delta \tau(z)\rangle=\sqrt{\frac{16 z L_{F}}{3 \pi}\left\langle b_{\omega}^{2}(z)\right\rangle}=\frac{8}{\omega L_{B}} \sqrt{\frac{\pi}{3} z L_{F}}
$$

where the last equality is obtained using (2).

The RMM is the only fiber model proposed to date that is consistent with polarization optical time-domain reflectometry results [12], [17]; however, we will show subsequently that its analysis is complex. For comparison, we consider also the FMM, which is simpler and provides the same results as the
RMM for the mean DGD in the case of unspun fibers [11]. According to the FMM, the local birefringence vector is

$$
\boldsymbol{\beta}(z, \omega)=b(\omega)(\cos 2 \theta(z), \sin 2 \theta(z), 0)^{T}
$$

where $\theta(z)$ is a Wiener process, i.e., it obeys the equation $d \theta / d z=\sigma_{F} \eta(z)$. In this expression, $\sigma_{F}$ is a constant parameter, and $\eta(z)$ is a white noise process [11]. According to this model, the birefringence correlation length and the beat length are $L_{F}=1 /\left(2 \sigma_{F}^{2}\right)$ and $L_{B}=2 \pi / b$, respectively.

The two models that we have introduced describe the random evolution of the intrinsic birefringence of a fiber without spin. When the spin is applied, the local birefringence vector undergoes a rotation. If we define a spin function $A(z)$, i.e., the angle of the rotation imposed on the fiber, then the birefringence vector of the spun fiber is $\mathbf{R}_{3}[2 A(z)] \beta(z)$, where

$$
\mathbf{R}_{3}(\phi)=\left(\begin{array}{ccc}
\cos \phi & -\sin \phi & 0 \\
\sin \phi & \cos \phi & 0 \\
0 & 0 & 1
\end{array}\right)
$$

represents a rotation of angle $\phi$ around the vector $(0,0,1)^{T}$. As we already stated, only periodic spin functions are considered in this paper, and hereafter $p$ represents the spin period, so that $A(z)$ is a $p$-periodic function.

\section{NUMERICAL COMPARISON}

In order to analyze the effect of spinning on the DGD of a fiber, it is useful to introduce the spin-induced reduction factor (SIRF) [8], which is the ratio between the mean DGD of a spun fiber and the mean DGD that the same fiber would have if it were not spun, as follows:

$$
\mathrm{SIRF}=\frac{\langle\Delta \tau(z)\rangle}{\left\langle\Delta \tau_{\mathrm{un}}(z)\right\rangle}
$$

where $\left\langle\Delta \tau_{\text {un }}(z)\right\rangle$ is the mean DGD of the unspun fiber.

We implement the RMM and the FMM and perform Monte Carlo simulations solving (1) for a set of 15000 fibers and for both birefringence models and different values of $L_{B}$ and $L_{F}$. For each value of $L_{F}$, we consider a fiber of length $z \geqslant 100 L_{F}$ so that the transient behavior has completely died out [18]. We then estimate the mean DGD, first for the unspun fiber and then for a fiber with the same birefringence parameters, which is spun using the sinusoidal function

$$
A(z)=A_{0} \sin \left(\frac{2 \pi}{p} z\right)
$$

There are four quantities that influence the mean DGD of a sinusoidally spun fiber- $L_{B}, L_{F}, p$, and $A_{0}$. In order to systematically consider a large portion of the possible regimes, we consider the two ratios: $p / L_{B}$ and $L_{F} / L_{B}$. Then, each of these ratios can be much smaller, of the same order, or much bigger than one.

We divide the remainder of this section into three parts, corresponding to $p / L_{B} \ll 1, p / L_{B} \simeq 1$, and $p / L_{B} \gg 1$, and for each of them, we vary the birefringence correlation length and the spin amplitude. Precisely, we varied $L_{F}$ uniformly from 0.5 m up to $100 \mathrm{~m}$, and $A_{0}$ from $0 \mathrm{rad} / \mathrm{m}$ up to $5 \mathrm{rad} / \mathrm{m}$ with a step 

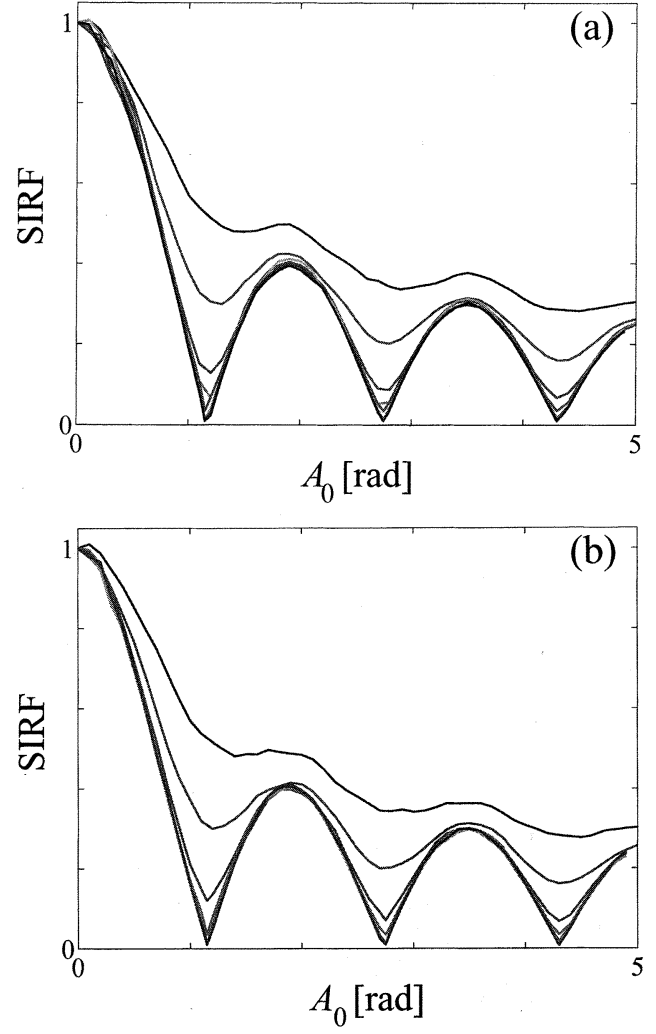

Fig. 1. Numerical estimates of the variation of the SIRF as a function of spin amplitude, for a sinusoidal spin function with $p=5 \mathrm{~m}$ and $L_{B}=21.3 \mathrm{~m}$. Plots (a) and (b) correspond to the FMM and the RMM, respectively. The solid curves, from the higher to the lower, refer to $L_{F}=1,2,5,10,20,50$, and 100 $\mathrm{m}$, respectively.

of $0.05 \mathrm{rad} / \mathrm{m}$; in this way, we considered both optimized and nonoptimized spin functions. In the following, we report the results obtained only for three pairs of values of $p$ and $L_{B}$, but we verified with further simulations that they represent the typical behavior of the three cases $p / L_{B} \ll 1, p / L_{B} \simeq 1$, and $p / L_{B} \gg 1$.

\section{A. First Regime: $p / L_{B} \ll 1$}

We first estimate the variation of the SIRF as a function of the spin amplitude for $p=5 \mathrm{~m}$ and $L_{B}=21 \mathrm{~m}$. For clarity, we report in Fig. 1(a) and (b) only a subset of the simulations. The curves, from the upper to the lower, are obtained for $L_{F}=1$, 2, 5, 10, 20, 50, and $100 \mathrm{~m}$. Fig. 1(a) corresponds to the results obtained with the FMM, and Fig. 1(b) shows the results obtained with the RMM.

We note that there is very good agreement between the two plots, confirming that the two models of birefringence produce the same results in the short-period limit. When $L_{F}$ is very short, the spin is not very effective in reducing the mean DGD for both the FMM and the RMM. However, as the correlation length increases, deep minima are evident in the SIRF plots. These minima correspond to those obtained in [7], for the case of polarization maintaining fibers $\left(L_{F} \rightarrow \infty\right)$. In addition, the curve that corresponds to $L_{F}=10 \mathrm{~m}$ is already very close to the curve for $L_{F}=100 \mathrm{~m}$. This result indicates that a spun fiber with a birefringence correlation length equal to only $10 \mathrm{~m}$
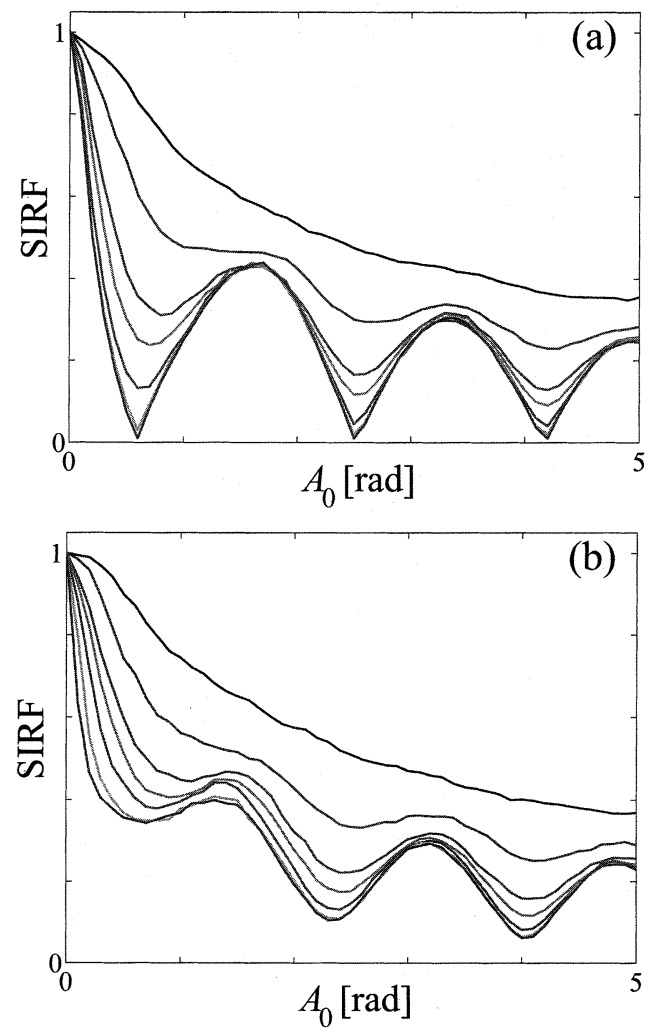

Fig. 2. Numerical estimates for the variation of the SIRF with the FMM as a function of spin amplitude, for a sinusoidal spin function with $p=4 \mathrm{~m}$ and $L_{B}=4.4 \mathrm{~m}$. Plots (a) and (b) correspond to the FMM and the RMM, respectively. The solid curves, from the higher to the lower, refer to $L_{F}=0.5$, $1,2,3,6,16$, and $50 \mathrm{~m}$, respectively.

is already very close to the deterministic limit and confirms that when $p<L_{B}$, the spin is able to give order to a randomly perturbed fiber.

The agreement between Fig. 1(a) and (b) also validates the expression presented in [8] for the mean DGD of a periodically spun randomly birefringent fiber in the short-period limit.

\section{B. Second Regime: $p / L_{B} \simeq 1$}

As a second case, we fix $p=4 \mathrm{~m}$ and $L_{B}=4.4 \mathrm{~m}$. The curves in Fig. 2(a) and (b), from the upper to the lower, are obtained for $L_{F}=0.5,1,2,3,16$, and $50 \mathrm{~m}$.

Fig. 2(a) corresponds to the results obtained with the FMM, and Fig. 2(b) reports the results obtained with the RMM, respectively. They show that there is a fairly good agreement between the two models only for the shortest values of $L_{F}$, when the spin is less effective. On the contrary, as the correlation length increases, the difference between the two models becomes evident. In particular, we note that in this regime the SIRF curves obtained with the FMM in Fig. 2(a) have marked local minima, whose values tend to zero as $L_{F}$ increases. Conversely, the SIRF curves obtained with the RMM in Fig. 2(b) have local minima whose values do not tend to zero, even for the largest values of $L_{F}$, and whose positions do not coincide to those obtained with the FMM. The difference between the results in these two models is due to the statistical variation of the birefringence value itself. If the birefringence strength is fixed in each step of the fiber, then the spinning may be able to exactly compensate 

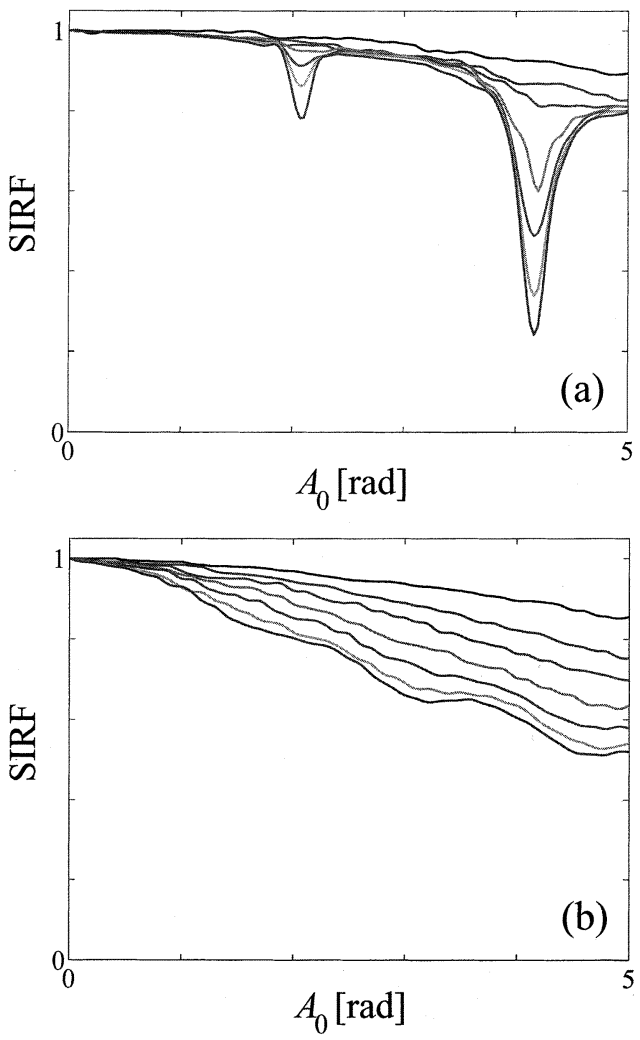

Fig. 3. Numerical estimates of the variation of the SIRF with the FMM as a function of spin amplitude, for a sinusoidal spin function with $p=18 \mathrm{~m} L_{B}=$ $4.4 \mathrm{~m}$. Plots (a) and (b) correspond to the FMM and the RMM, respectively. The solid curves, from the higher to the lower, refer to $L_{F}=1,3,5,10,18,30$ and $50 \mathrm{~m}$, respectively.

for it, whereas in the case of randomly varying birefringence strength, exact compensation is never possible.

\section{Third Regime: $p / L_{B} \gg 1$}

As a third case, we fix $p=18 \mathrm{~m}$ and $L_{B}=4.4 \mathrm{~m}$. The solid curves, from the upper to the lower, in Fig. 3(a) and (b) correspond to the SIRF function for the following values of the correlation length: $L_{F}=1,3,5,10,18,30$, and $50 \mathrm{~m}$.

From Fig. 3(a) and (b), which refers to the FMM and the RMM, respectively, it can be seen that in this case the spin is not effective in reducing the mean DGD. Moreover, the two models only agree for the shortest values of $L_{F}$. Also in this regime, the main difference between the FMM and the RMM is that, as soon as $L_{F} \geqslant L_{B}$, the SIRF has marked minima in the FMM that do not appear when the RMM is used.

\section{Summary of the Comparison}

Table I shows the comparison between the FMM and the RMM in the regimes we have identified. We note that the two models only consistently agree in the short-period limit.

This result confirms that the formula obtained in [8] for the mean DGD of a periodically spun fiber holds well for both birefringence models when the spin period is shorter than the beat length. Conversely, when the spin period is of the same order or bigger than the beat length, the simpler analysis that can be
TABLE I

COMPARISON BETWEEN THE FMM AND THE RMM

\begin{tabular}{c|c|c}
\hline \hline$p / L_{B}$ & $L_{F} / L_{B}$ & FMM vs. RMM \\
\hline \multirow{3}{*}{$\ll 1$} & $\ll 1$ & Agree \\
& $\simeq 1$ & Agree \\
& $\gg 1$ & Agree \\
\hline & $\ll 1$ & Agree \\
$\simeq 1$ & $\simeq 1$ & Disagree \\
& $\gg 1$ & Disagree \\
\hline \multirow{3}{*}{$\gg 1$} & $\ll 1$ & Agree \\
& $\simeq 1$ & Disagree \\
& $\gg 1$ & Disagree \\
\hline \hline
\end{tabular}

performed by means of the FMM leads to a mean DGD that differs significantly from the mean DGD that is obtained with the RMM. Therefore, the behavior of randomly birefringent, periodically spun fibers differs significantly from the case of unspun fibers, where the mean DGD is independent of the model used for the random birefringence [11].

In addition, in Figs. 1-3, we note that the mean DGD of a spun fiber decreases as $L_{F}$ increases, a result that also differs from the case of unspun fibers.

Moreover, it has only recently become possible to measure the local birefringence of optical fibers, and it is even more difficult to predict its value before drawing a fiber. As a consequence, it is reasonable that a large number of spun fibers may have a spin period of the same order as or even longer than their beat length. Therefore, it is necessary to analyze spun fibers using the RMM.

\section{THEORY ON SPUN FibERS With THE RMM}

The analysis of periodically spun fibers using the RMM can be performed following the same procedure that was used for the FMM [8], [19]. We start by transforming the reference frame in order to compensate for both the intrinsic rotation of the birefringence and the rotation induced by the spin, using the matrix $\mathbf{T}(z)=\mathbf{R}_{3}^{T}[2(A(z)+\theta(z))]$ [7]. The angle $\theta(z)$ in the RMM is defined in a similar way as in the FMM, i.e., considering $\beta_{1}(z)=b(z) \cos 2 \theta(z)$ and $\beta_{2}=b(z) \sin 2 \theta(z)$ for the unspun fiber. In this case, however, $b(z)=\left(\beta_{1}^{2}+\beta_{2}^{2}\right)^{1 / 2}$ is a random process. If we indicate with $\boldsymbol{\Omega}_{f}$ and $\boldsymbol{\beta}_{f}$ the polarization dispersion vector and the birefringence vector in the fixed reference frame, then the change of coordinate system is implemented by $\boldsymbol{\Omega}(z)=\mathbf{T}(z) \boldsymbol{\Omega}_{f}(z)$, and $\boldsymbol{b}(z)=\mathbf{T}(z) \boldsymbol{\beta}_{f}(z)$. The dynamical equation in the rotating reference frame can be obtained after calculating the matrix $d \mathbf{T} / d z$; therefore, we must calculate the $z$ derivative of $\theta(z)$ and we find that

$$
\frac{d \theta}{d z}=\frac{\sigma}{2 b^{2}}\left(\eta_{2} \beta_{1}-\eta_{1} \beta_{2}\right) .
$$

Finally, the dynamical equation in the new reference frame reads

$$
\partial \boldsymbol{\Omega} / \partial z=\left(\begin{array}{c}
b \\
0 \\
-2 \alpha-\sigma\left(\eta_{2} \beta_{1}-\eta_{1} \beta_{2}\right) / b^{2}
\end{array}\right) \times \boldsymbol{\Omega}+\left(\begin{array}{c}
b / \omega \\
0 \\
0
\end{array}\right)
$$


where $\alpha(z)$ is the $z$ derivative of $A(z)$. The matrix $\mathbf{T}(z)$ is orthogonal so that the modulus of $\boldsymbol{\Omega}(z)$ still equals the DGD. Equations (8) and (3) form a system of stochastic differential equations that can be rewritten as

$\frac{d}{d z}\left(\begin{array}{c}\Omega_{1} \\ \Omega_{2} \\ \Omega_{3} \\ \beta_{1} \\ \beta_{2}\end{array}\right)$

$=\frac{\sigma}{b^{2}}\left(\begin{array}{cc}-\beta_{2} \Omega_{2} & \beta_{1} \Omega_{2} \\ \beta_{2} \Omega_{1} & -\beta_{1} \Omega_{1} \\ 0 & 0 \\ b^{2} & 0 \\ 0 & b^{2}\end{array}\right)\left(\begin{array}{l}\eta_{1} \\ \eta_{2}\end{array}\right)+\left(\begin{array}{c}2 \alpha \Omega_{2}+\frac{b}{\omega} \\ -b \Omega_{3}-2 \alpha \Omega_{1} \\ b \Omega_{2} \\ -\rho \beta_{1} \\ -\rho \beta_{2}\end{array}\right)$.

By means of the theory of stochastic differential equations [20], we calculate the infinitesimal generator $\mathcal{A}$ associated with (9), and using Dynkin's formula, we obtain an equation for the evolution of the mean-square DGD

$$
\frac{d\left\langle\Delta \tau^{2}\right\rangle}{d z}=\frac{2}{\omega}\left\langle b \Omega_{1}\right\rangle
$$

Using Dynkin's formula again, we find

$$
\begin{aligned}
\frac{d\left\langle b \Omega_{1}\right\rangle}{d z} & =\frac{\left\langle b^{2}\right\rangle}{\omega}+2 \alpha\left\langle b \Omega_{2}\right\rangle-\rho\left\langle b \Omega_{1}\right\rangle \\
\frac{d\left\langle b \Omega_{2}\right\rangle}{d z} & =-2 \alpha\left\langle b \Omega_{1}\right\rangle-\rho\left\langle b \Omega_{2}\right\rangle-\left\langle b^{2} \Omega_{3}\right\rangle \\
\frac{d\left\langle b^{2} \Omega_{3}\right\rangle}{d z} & =-2 \rho\left\langle b^{2} \Omega_{3}\right\rangle+2 \sigma^{2}\left\langle\Omega_{3}\right\rangle+\left\langle b^{3} \Omega_{2}\right\rangle .
\end{aligned}
$$

The iterative application of the generator leads to an infinite sequence of relationships that can be expressed recursively as

$$
\begin{aligned}
\frac{d\left\langle b^{2 h} \Omega_{3}\right\rangle}{d z}= & 2 h^{2} \sigma^{2}\left\langle b^{2(h-1)} \Omega_{3}\right\rangle-2 \rho h\left\langle b^{2 h} \Omega_{3}\right\rangle+\left\langle b^{2 h+1} \Omega_{2}\right\rangle \\
\frac{d\left\langle b^{2 h+1} \Omega_{1}\right\rangle}{d z}= & \frac{\left\langle b^{2(h+1)}\right\rangle}{\omega}+2 \sigma^{2} h(h+1)\left\langle b^{2(h-1)+1} \Omega_{1}\right\rangle \\
& -(2 h+1) \rho\left\langle b^{2 h+1} \Omega_{1}\right\rangle+2 \alpha\left\langle b^{2 h+1} \Omega_{2}\right\rangle \\
\frac{d\left\langle b^{2 h+1} \Omega_{2}\right\rangle}{d z}= & 2 h(h+1) \sigma^{2}\left\langle b^{2(h-1)+1} \Omega_{2}\right\rangle-2 \alpha\left\langle b^{2 h+1} \Omega_{1}\right\rangle \\
& -\rho(2 h+1)\left\langle b^{2 h+1} \Omega_{2}\right\rangle-\left\langle b^{2(h+1)} \Omega_{3}\right\rangle \quad(11)
\end{aligned}
$$

with initial conditions $\left\langle b^{2 h} \Omega_{3}\right\rangle=\left\langle b^{2 h+1} \Omega_{1}\right\rangle=\left\langle b^{2 h+1} \Omega_{2}\right\rangle=0$ at $z=0$ for all $h$.

Before proceding with the analysis, we note that (11) is an infinite system of equations due to the correlation between the two variables $b^{2}$ and $\Omega_{3}$. If they were uncorrelated, it would be possible to obtain the same three-dimensional system as was obtained for the FMM. For unspun fibers, it is reasonable to assume that these two variables are uncorrelated as was shown in [21] and is suggested by numerical simulations. Conversely, numerical simulations show that it is not at all straightforward to extend this property to the case of spun fibers.

The set of (11) is unlikely to be solved explicitly due to its evident complexity. To obtain a numerical solution, it must be possible to truncate the infinite-dimensional system. If the sequence that we obtained in (11) does not converge, then truncation is not possible, and one must solve the original stochastic differential equation. We will find that in some cases the sequence converges and a numerical solution is possible, but in other cases, it does not converge.

We note that in (11), the forcing term increases as $h$ ! because $\left\langle b^{2 h}\right\rangle=h !\left(2 \sigma_{\beta}^{2 h}\right)$. Therefore, it is convenient to introduce a change of variables; we choose

$$
\begin{aligned}
& y_{0}(z, h)=\frac{1}{h ! h !\left(2 \sigma_{\beta}^{2}\right)^{h}}\left\langle b^{2 h} \Omega_{3}\right\rangle \\
& y_{1}(z, h)=\frac{1}{h !(h+1) !\left(2 \sigma_{\beta}^{2}\right)^{h}}\left\langle b^{2 h+1} \Omega_{1}\right\rangle \\
& y_{2}(z, h)=\frac{1}{h !(h+1) !\left(2 \sigma_{\beta}^{2}\right)^{h}}\left\langle b^{2 h+1} \Omega_{2}\right\rangle
\end{aligned}
$$

where we recall that $\sigma_{\beta}^{2}=\left\langle b^{2}\right\rangle=\sigma^{2} / 2 \rho$. Using (11), and (12), we find

$$
\begin{aligned}
\frac{d y_{0}(z, h)}{d z}= & 2 \rho\left(1-\delta_{h, 0}\right) y_{0}(z, h-1) \\
& -2 \rho h y_{0}(z, h)+(h+1) y_{2}(z, h) \\
\frac{d y_{1}(z, h)}{d z}= & 2 \rho\left(1-\delta_{h, 0}\right) y_{1}(z, h-1) \\
& -(2 h+1) \rho y_{1}(z, h)+2 \alpha y_{2}(z, h)+\frac{2 \sigma_{\beta}^{2}}{h ! \omega} \\
\frac{d y_{2}(z, h)}{d z}= & 2 \rho\left(1-\delta_{h, 0}\right) y_{2}(z, h-1)-2 \alpha y_{1}(z, h) \\
& -(2 h+1) \rho y_{2}(z, h)-2 \sigma_{\beta}^{2}(h+1) y_{0}(z, h+1)
\end{aligned}
$$

where $\delta_{h, 0}$ is the Kronecker delta. Equation (13) can be rearranged as follows: we define the vector $\boldsymbol{y}(z)$, whose $(3 h+n)$ th element equals $y_{n}(z, h)$ with $h \geqslant 0$ and $n=0,1,2$. According to (13), $\boldsymbol{y}$ obeys the equation

$$
\frac{d \boldsymbol{y}}{d z}=\mathbf{A}(z) \boldsymbol{y}(z)+\boldsymbol{u}
$$

where $\mathbf{A}(z)$ is a $z$-dependent infinite-dimensional matrix, and $\boldsymbol{u}$ is a constant infinite-dimensional vector, given by (15), shown at the bottom of the next page. Equation (14) is an inhomogeneous infinite-dimensional system with periodic coefficients and with the initial condition $\boldsymbol{y}(0)=0$. We note that (14) is very similar to the system obtained studying the twist induced circular birefringence in long single-mode fibers [16]. Analogous to what was reported in [16] and using the same approach, it is possible to prove that the following properties hold:

$$
\begin{aligned}
& \left|y_{0}(z ; h)\right| \leqslant \frac{2^{h}}{h !} \sqrt{\left\langle\Omega_{3}^{2}\right\rangle} \\
& \left|y_{1}(z ; h)\right| \leqslant \frac{2^{h}}{h !} \sqrt{\left\langle\left(b^{2} \Omega_{1}^{2}\right)^{2}\right\rangle} \\
& \left|y_{2}(z ; h)\right| \leqslant \frac{2^{h}}{h !} \sqrt{\left\langle\left(b^{2} \Omega_{2}^{2}\right)^{2}\right\rangle}
\end{aligned}
$$

which state that, as $h$ increases, the modulus of $y_{n}(z ; h)$ decreases very quickly. The proof is given in the Appendix. Moreover, the elements of A grow linearly with $h$ so that the modulus of the derivative of $y_{n}(z ; h)$ decreases quickly. As a consequence, it seems reasonable to truncate the recursive equations. We set $y_{n}(z, h)=0$ for every $z \geqslant 0$ and for every $h \geqslant H$, 
with $H$ sufficiently large. By means of this approximation, (14) becomes a system of differential equations of dimension $3 H$, which can be numerically integrated. Equation (10) gives an expression for the growth of the mean-square DGD in terms of $\left\langle b \Omega_{1}\right\rangle$, which is proportional to $y_{1}(z, 0)$. Note that even though we are interested only in the second component $y_{1}(z, 0)$, we have to solve the whole system. We also emphasize that there is no guarantee that this truncation will converge as $H$ increases.

We solved the system (14), truncated at various values of $H$ using both MATLAB and the CVODE differential equation package [22]. We also exploited sparse matrix methods since the matrix is banded with dimension 6 . We then compared this solution for the mean-square DGD with the mean values obtained by Monte Carlo numerical simulations, as described in the previous section. We used this procedure for a sinusoidal spin function with several values of $L_{B}, L_{F}, p$, and $A_{0}$. In general, the solution of the truncated system is much faster than the Monte Carlo simulations, but the truncation does not always converge. We found that when $L_{F}$ approaches or is bigger than $L_{B}$, truncating the system gives incorrect results.

We have found empirically that the solution of the truncated system yields correct results with $H$ as small as 20 when both $L_{F}$ and $p$ are smaller or of the same order as $L_{B}$ and when $A_{0}$ is a few radians. When the spin amplitude is larger, we found that it is necessary to truncate the system at a larger $H$.

\section{Perturbative AnAlysis}

The analysis performed in the previous section allows one to gain partial information on the behavior of spun fibers according to the RMM. Another approach to the problem is to use perturbative methods [23], [24]. These methods are applicable when one system parameter is much smaller than another, and consequently, we will consider the case of a short spin period and the case of a short correlation length.

For convenience, we rewrite here the first three equations of the infinite-dimensional system

$$
\begin{aligned}
\frac{d\left\langle b \Omega_{1}\right\rangle}{d z} & =\frac{\left\langle b^{2}\right\rangle}{\omega}+2 \alpha\left\langle b \Omega_{2}\right\rangle-\rho\left\langle b \Omega_{1}\right\rangle \\
\frac{d\left\langle b \Omega_{2}\right\rangle}{d z} & =-2 \alpha\left\langle b \Omega_{1}\right\rangle-\rho\left\langle b \Omega_{2}\right\rangle-\left\langle b^{2} \Omega_{3}\right\rangle \\
\frac{d\left\langle b^{2} \Omega_{3}\right\rangle}{d z} & =2 \sigma^{2}\left\langle\Omega_{3}\right\rangle-2 \rho\left\langle b^{2} \Omega_{3}\right\rangle+\left\langle b^{3} \Omega_{2}\right\rangle .
\end{aligned}
$$

\section{A. Case of a Short Spin Period}

We consider the case in which the spin period is shorter than the beat length and the correlation length. Under this condition, the leading order terms of the asymptotic expansions for $\left\langle b \Omega_{1}\right\rangle$, $\left\langle b \Omega_{2}\right\rangle$, and $\left\langle b^{2} \Omega_{3}\right\rangle$ are

$$
\begin{aligned}
\frac{d\left\langle b \Omega_{1}\right\rangle^{(0)}}{d z} & =\frac{\left\langle b^{2}\right\rangle}{\omega}+2 \alpha\left\langle b \Omega_{2}\right\rangle^{(0)} \\
\frac{d\left\langle b \Omega_{2}\right\rangle^{(0)}}{d z} & =-2 \alpha\left\langle b \Omega_{1}\right\rangle^{(0)} \\
\frac{d\left\langle b^{2} \Omega_{3}\right\rangle^{(0)}}{d z} & =-2 \alpha\left\langle b \Omega_{1}\right\rangle^{(0)} .
\end{aligned}
$$

We note that the first two equations are coupled, and along with the initial conditions $\left\langle b \Omega_{1}\right\rangle^{(0)}=\left\langle b \Omega_{2}\right\rangle^{(0)}=0$ at $z=0$, (18) can be solved by means of variation of parameters. By substituting

$$
\begin{aligned}
\left\langle b \Omega_{1}(z)\right\rangle^{(0)} & =k_{1}(z) \sin [2 A(z)]+k_{2}(z) \cos [2 A(z)] \\
\left\langle b \Omega_{2}(z)\right\rangle^{(0)} & =-k_{2}(z) \sin [2 A(z)]+k_{1}(z) \cos [2 A(z)]
\end{aligned}
$$

we find that

$$
\begin{aligned}
& k_{1}(z)=\frac{\left\langle b^{2}\right\rangle}{\omega} \int_{0}^{z} \sin [2 A(z)] \equiv \frac{\left\langle b^{2}\right\rangle}{\omega} S \\
& k_{2}(z)=\frac{\left\langle b^{2}\right\rangle}{\omega} \int_{0}^{z} \cos [2 A(z)] \equiv \frac{\left\langle b^{2}\right\rangle}{\omega} C
\end{aligned}
$$

from which we conclude that

$$
\left\langle\Delta \tau^{2}\right\rangle=\frac{\left\langle b^{2}\right\rangle}{\omega^{2}}\left(C^{2}+S^{2}\right) .
$$

Recalling that $\left\langle b^{2}\right\rangle=2 \sigma_{\beta}^{2}=4 \pi^{2} / L_{B}^{2}$, we find that (21) coincides with the expression obtained in this regime with the FMM [25], confirming the agreement of the two models in the case of fast spin.

\section{B. Case of a Short Correlation Length}

We analyze by means of the perturbative technique, the regime $L_{F} \ll L_{B}, L_{F} \ll p$, and spin amplitudes of a few ra-

$$
\begin{aligned}
& \mathbf{A}=\left(\begin{array}{cccccccccc}
0 & 0 & +1 & 0 & \ldots & & & & & \\
0 & -\rho & +2 \alpha & 0 & 0 & \ldots & & & & \\
0 & -2 \alpha & -\rho & -2 \sigma_{\beta}^{2} & 0 & 0 & \ldots & & & \\
+2 \rho & 0 & 0 & -2 \rho & 0 & +2 & 0 & \ldots & & \\
0 & +2 \rho & 0 & 0 & -3 \rho & +2 \alpha & 0 & 0 & \ldots & \\
\ldots & 0 & +2 \rho & 0 & -2 \alpha & -3 \rho & -4 \sigma_{\beta}^{2} & 0 & 0 & \ldots \\
& \ldots & 0 & +2 \rho & 0 & 0 & -4 \rho & 0 & +3 & \ldots \\
& & \ldots & 0 & +2 \rho & 0 & 0 & -5 \rho & +2 \alpha & \ldots \\
& & & & \ldots & +2 \rho & 0 & -2 \alpha & -5 \rho & -6 \sigma_{\beta}^{2} \ldots
\end{array}\right) \\
& \boldsymbol{u}=\left(\begin{array}{llllllllll}
0 & 2 \sigma_{\beta}^{2} / \omega & 0 & 0 & 2 \sigma_{\beta}^{2} /(1 ! \omega) & 0 & 0 & 2 \sigma_{\beta}^{2} /(2 ! \omega) & 0 & \ldots
\end{array}\right)^{T} \\
&
\end{aligned}
$$


dians. The leading-order equations in the asymptotic expansion are

$$
\begin{aligned}
\frac{d\left\langle b \Omega_{1}\right\rangle^{(0)}}{d z} & =\frac{\left\langle b^{2}\right\rangle}{\omega}-2 \rho\left\langle b \Omega_{1}\right\rangle^{(0)} \\
\frac{d\left\langle b \Omega_{2}\right\rangle^{(0)}}{d z} & =-2 \rho\left\langle b \Omega_{2}\right\rangle^{(0)} \\
\frac{d\left\langle b^{2} \Omega_{3}\right\rangle^{(0)}}{d z} & =-2 \rho\left\langle b^{2} \Omega_{3}\right\rangle^{(0)} .
\end{aligned}
$$

Using the initial conditions $\left\langle b \Omega_{1}\right\rangle^{(0)}=\left\langle b \Omega_{2}\right\rangle^{(0)}=$ $\left\langle b^{2} \Omega_{3}\right\rangle^{(0)}=0$, the leading-order solution is

$$
\begin{aligned}
\left\langle b \Omega_{1}\right\rangle^{(0)} & =\frac{\left\langle b^{2}\right\rangle}{2 \rho \omega}[1-\exp (-2 \rho z)] \\
\left\langle b \Omega_{2}\right\rangle^{(0)} & =0 \\
\left\langle b^{2} \Omega_{3}\right\rangle^{(0)} & =0 .
\end{aligned}
$$

Substituting the solution for $\left\langle b \Omega_{1}\right\rangle^{(0)}$ in the expression for the mean DGD, we obtain the same solution as for an unspun fiber. The correction at the next order is

$$
\begin{aligned}
& \frac{d\left\langle b \Omega_{1}\right\rangle^{(1)}}{d z}=-\rho\left\langle b \Omega_{1}\right\rangle^{(1)} \\
& \frac{d\left\langle b \Omega_{2}\right\rangle^{(1)}}{d z}=-2 \alpha\left\langle b \Omega_{1}\right\rangle^{(0)}-2 \rho\left\langle b \Omega_{2}\right\rangle^{(1)} .
\end{aligned}
$$

Consequently, at this order, there is no correction to $\left\langle b \Omega_{1}\right\rangle$. Solving the equation for $\left\langle b \Omega_{2}\right\rangle^{(1)}$, we introduce the spin function $A(z)=A_{0} \sin (2 \pi z / p)$, and we obtain

$$
\begin{array}{r}
\left\langle b \Omega_{2}\right\rangle^{(1)}=-\frac{2 A_{0} \nu\left\langle b^{2}\right\rangle}{\omega \rho\left(\nu^{2}+\rho^{2}\right)}\left\{\nu \rho \cos (\nu z)+\nu^{2} \sin (\nu z)\right. \\
\left.-\exp (-\rho z)\left[\rho \nu+\left(\nu^{2}+\rho^{2}\right) \sin (\nu z)\right]\right\}
\end{array}
$$

where $\nu=2 \pi / p$. Next, the equation for the second-order correction is

$$
\frac{d\left\langle b \Omega_{1}\right\rangle^{(2)}}{d z}=-\rho\left\langle b \Omega_{1}\right\rangle^{(2)}+2 \alpha\left\langle b \Omega_{2}\right\rangle^{(1)}
$$

and we find

$$
\begin{array}{r}
\left\langle b \Omega_{1}\right\rangle^{(2)}=-\frac{2 A_{0}^{2} \nu^{2}\left\langle b^{2}\right\rangle}{\omega \rho\left(\rho^{2}+\nu^{2}\right)\left(\rho^{2}+4 \nu^{2}\right)}\left\{\rho^{2}[1+\cos (2 \nu z)]+4 \nu^{2}\right. \\
\left.+2 \rho \nu \sin (2 \nu z)-2\left(\rho^{2} \nu^{2}\right) \exp (-\rho z)\right\} .
\end{array}
$$

Finally, the sum $\left\langle b \Omega_{1}\right\rangle^{(0)}+\left\langle b \Omega_{1}\right\rangle^{(2)}$ can be integrated over distance to obtain the mean-square DGD, according to (10), yielding the expression

$$
\left\langle\Delta \tau^{2}\right\rangle \simeq\left\langle\Delta \tau_{\mathrm{un}}^{2}\right\rangle\left(1-\frac{2 A_{0} \nu^{2}}{\rho^{2}+\nu^{2}}\right)
$$

This expression was simplified under the condition $z \gg L_{F}$. Moreover, it coincides with that obtained for the FMM in [25], and it has the same range of validity. In particular, it can be noted that the factor in parentheses is always positive under the conditions $L_{F} \ll L_{B}, L_{F} \ll p$, and the spin amplitudes of a few radians. In addition, we have verified that if $p /\left(2 \pi A_{0}\right) \geqslant$
$6 L_{F}$ and $p \simeq L_{B}$, (26) differs less than $5 \%$ from numerical simulation results. If $p<L_{B}$, the error is less than $5 \%$ for even smaller values of $p /\left(2 \pi A_{0}\right)$ with respect to $L_{F}$.

\section{CONCLUSION}

We carried out an extensive study of the effects of a periodic spin on the PMD of randomly perturbed fibers, considering the two Wai-Menyuk models of random birefringence.

Numerical simulations show that spinning is more effective in reducing the mean DGD of a fiber when its period is shorter than the beat length. In this case, the two Wai-Menyuk models yield the same results. Conversely, when the spin period is of the same order as or greater than the beat length, the mean DGD of a periodically spun fiber strongly depends on the model used for the random birefringence. This result contrasts with previous results for unspun fibers.

Hence, we carried out a theoretical analysis with the random modulus model, which is the only model consistent with experimental data. The result of this study is an infinite-dimensional system of differential equations that can be solved numerically by truncation and yields the average behavior of the DGD with less computational time than Monte Carlo simulations when it converges. However, the truncation does not always converge, and we found that the system cannot be truncated when $L_{F}$ is of the same order or greater than $L_{B}$. One may object that truncation works in the same regimes where the RMM and the FMM do provide the same mean DGD. Yet, we believe that the derivation of the infinite-dimensional system presented in this paper is the starting point for any analytical treatment of periodically spun fibers with the RMM, and its knowledge may open the way to other researchers for a solution of wider validity.

Finally, we studied periodically spun fibers using the RMM by means of perturbative techniques in the case of a short spin period and of a short birefringence correlation length. In both of these cases, we have been able to find an analytical formula for the mean DGD with the RMM.

\section{APPENDIX}

Let $v$ and $w$ be two real random variables. By means of the Schwarz inequality, it is possible to prove the property

$$
\mathrm{E}[v w]^{2} \leqslant \mathrm{E}\left[v^{2}\right] \mathrm{E}\left[w^{2}\right] .
$$

Applying (27) to $y_{n}(z ; h),(n=0,1,2)$, we obtain (16). Here, we report the demonstration for $y_{0}(z ; h)$; the same procedure can be followed for $y_{1}(z ; h)$ and $y_{2}(z ; h)$. We recall that

$$
y_{0}(z, h)=\frac{1}{(h !)^{2}\left(2 \sigma_{\beta}^{2}\right)^{h}}\left\langle b^{2 h} \Omega_{3}\right\rangle .
$$

As a consequence, we may write

$$
\begin{aligned}
y_{0}(z, h)^{2}= & \frac{1}{(h !)^{4}\left(2 \sigma_{\beta}^{2}\right)^{2 h}}\left\langle b^{2 h} \Omega_{3}\right\rangle^{2} \\
& \leqslant \frac{1}{(h !)^{4}\left(2 \sigma_{\beta}^{2}\right)^{2 h}}\left\langle b^{4 h}\right\rangle\left\langle\Omega_{3}^{2}\right\rangle \\
= & \frac{(2 h) !\left\langle\Omega_{3}^{2}\right\rangle}{h !^{4}} .
\end{aligned}
$$


Now we would like to study the behavior of the term $\sqrt{(2 h) !} / h !^{2}$. It is possible to verify inductively that $\sqrt{(2 h) !} \leqslant 2^{h} h$ ! and $\sqrt{(2 h+1) !} \leqslant 2^{h}(h+1)$ !. Moreover the factorial can be approximated using Stirling formula $h ! \simeq h^{h} \sqrt{2 \pi h} \exp (-h)$. Hence, we find

$$
\frac{\sqrt{(2 h) !}}{h !^{2}} \simeq \frac{2^{h}}{h !} \simeq \frac{1}{\sqrt{2 \pi h}\left(\frac{h}{2 e}\right)^{h}}
$$

which tends to zero very rapidly.

\section{ACKNOWLEDGMENT}

This research was carried out in the framework of the agreement between Università di Padova and Istituto Superiore delle Comunicazioni e Tecnologie dell'Informazione. The authors would like to acknowledge the Laboratory for Physical Sciences, College Park, MD; the Laboratory for Telecommunications Sciences, Adelphi, MD; as well as the U.S. Department of Energy and National Science Foundation.

\section{REFERENCES}

[1] C. D. Poole and J. Nagel, "Polarization effects in lightwave systems," in Optical Fiber Telecommunications, I. P. Kaminow and T. Koch, Eds. San Diego, CA: Academic, 1997.

[2] M. A. Santoro and C. D. Poole, "Polarization scrambling using a short piece of high-birefringence optical fiber and a multifrequency laser diode," J. Lightwave Technol., vol. 12, pp. 288-293, Feb. 1994.

[3] H. Sunnerud, C. Xie, M. Karlsson, R. Samuelsson, and P. A. Andrekson, "A comparison between different PMD compensation techniques," $J$. Lightwave Technol., vol. 20, pp. 368-378, Mar. 2002.

[4] A. J. Barlow, J. J. Ramskov-Hansen, and D. N. Payne, "Birefringence and polarization mode-dispersion in spun single-mode fibers," Appl. Opt., vol. 20, pp. 2962-2968, 1981.

[5] A. Hart, R. G. Huff, and K. L. Walker, "Method of making a fiber having low polarization mode dispersion due to a permanent spin," U.S. Patent 5298 047, Mar. 29, 1994

[6] - "Article comprising optical fiber having low polarization mode dispersion, due to permanent spin," U.S. Patent 5418881, May 23, 1995.

[7] A. Galtarossa, L. Palmieri, and A. Pizzinat, "Optimized spinning design for low PMD fibers: An analytical approach," J. Lightwave Technol., vol. 19, pp. 1502-1512, Oct. 2001.

[8] A. Galtarossa, P. Griggio, A. Pizzinat, and L. Palmieri, "Calculation of mean differential group delay of periodically spun randomly birefringent fibers," Opt. Lett., vol. 27, pp. 692-694, 2002.

[9] X. Chen, M.-J. Li, and D. A. Nolan, "Polarization mode dispersion of spun fibers: An analytical solution," Opt. Lett., vol. 27, pp. 294-296, 2002.

[10] R. E. Schuh, X. Shan, and A. S. Siddiqui, "Polarization mode dispersion in spun fibers with different linear birefringence and spinning parameters," J. Lightwave Technol., vol. 16, pp. 1583-1588, Sept. 1998.

[11] P. K. A. Wai and C. R. Menyuk, "Polarization mode dispersion, decorrelation, and diffusion in optical fibers with randomly varying birefringence," J. Lightwave Technol., vol. 14, pp. 148-157, Feb. 1996.

[12] A. Galtarossa, L. Palmieri, M. Schiano, and T. Tambosso, "Statistical characterization of fiber random birefringence," OSA Opt. Lett., vol. 25, pp. 1322-1324, 2000.

[13] —, "Measurements of beat length and perturbation length in long single-mode fibers," OSA Opt. Lett., vol. 25, pp. 384-386, 2000.

[14] A. Galtarossa and L. Palmieri, "PMD in single-mode fibers: Measurments of local birefringence correlation length," in Tech. Digest. OFC, Anaheim, CA, 2002, Paper WA4.
[15] C. D. Poole, J. H. Winters, and J. A. Nagel, "Dynamical equation for polarization dispersion," Opt. Lett., vol. 6, pp. 372-374, 1991.

[16] A. Galtarossa and L. Palmieri, "Measure of twist-induced circular birefringence in long single-mode fibers: Theory and experiments," J. Lightwave Technol., vol. 20, pp. 1149-1159, July 2002.

[17] A. Galtarossa, L. Palmieri, M. Schiano, and T. Tambosso, "Measurement of birefringence correlation length in long single-mode fibers," OSA Opt. Lett., vol. 26, pp. 962-964, 2001.

[18] Y. Tan, J. Yang, W. L. Kath, and C. R. Menyuk, "Transient evolution of the polarization-dispersion vector's probability distribution," J. Opt. Soc. Amer. A, vol. 19, pp. 992-1000, 2002.

[19] A. Galtarossa, L. Palmieri, A. Pizzinat, B. S. Marks, and C. R. Menyuk, "An analytical formula for the mean differential group delay of randomly-birefringent spun fibers," J. Lightwave Technol., vol. 21, pp. 1635-1643, July 2002.

[20] B. Øksendal, Stochastic Differential Equations. Berlin, Germany: Springer-Verlag, 2000.

[21] C. R. Menyuk and P. K. A. Wai, "Polarization evolution and dispersion in fibers with spatially varying birefringence," J. Opt. Soc. Amer. B, vol. 11, pp. 1288-1296, 1994.

[22] S. D. Cohen and A. C. Hindmarsh. (1994) CVODE User Guide, Livermore. [Online]. Available: www.netlib.org

[23] A. H. Nayfeh, Perturbation Methods. New York: Wiley, 1973.

[24] J. Kevorkian and J. D. Cole, Perturbation Methods in Applied Mathematics. New York: Springer-Verlag, 1981.

[25] A. Pizzinat, B. S. Marks, L. Palmieri, C. R. Menyuk, and A. Galtarossa, "Polarization mode dispersion of spun fibers with randomly varying birefringence," Opt. Lett., vol. 28, pp. 390-392, 2003.

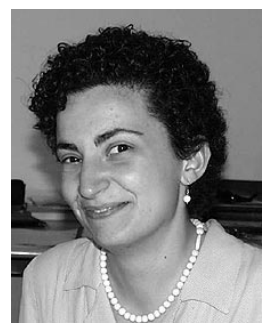

Anna Pizzinat was born in Treviso, Italy, in 1974. She received the Master's degree in electronic engineering and the Ph.D. degree from the University of Padova, Padova, Italy, in 1999 and 2003, respectively.

In 2002, she spent six months at the University of Maryland Baltimore County as a Visiting Student. She currently works in the Department of Information Enginering, University of Padova. Her research interests include the study of fibers with low polarization-mode dispersion, and the analysis and design of high bit-rate optical transmission systems.

Dr. Pizzinat received the Best Young Researcher Award from the Department of Information Enginering, University of Padova, in April 2003.

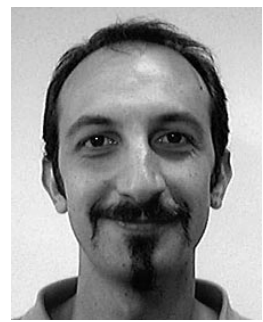

Luca Palmieri was born in Belluno, Italy, in 1971. $\mathrm{He}$ received the degree in electronic engineering and the Ph.D. degree from the University of Padova, Padova, Italy, in 1996 and 2000, respectively.

From 2000 to 2002, he was a Research Fellow at the University of Udine, Italy, and presently he is a Research Fellow at the University of Padova. His research activity mainly focuses on polarization-mode dispersion (PMD) causes and effects and spans from birefringence and PMD measurements techniques to PMD compensation and design of low-PMD optical

fibers.

Brian S. Marks, photograph and biography not available at the time of publication. 


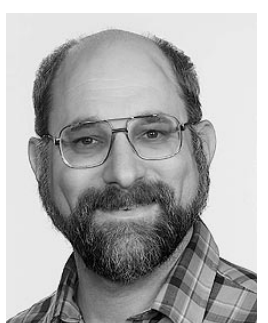

Curtis R. Menyuk (F'98) was born on March 26, 1954. He received the B.S. and M.S. degrees from the Massachusetts Institute of Technology (MIT), Cambridge, in 1976 and the Ph.D. degree from the University of California at Los Angeles (UCLA) in 1981.

He has worked as a Research Associate at the University of Maryland, College Park, and at Science Applications International Corporation, McLean, VA. In 1986, he became an Associate Professor in the Department of Electrical Engineering at the University of Maryland Baltimore County (UMBC) and he was the founding member of this department. In 1993, he was promoted to Professor. He was on partial leave from UMBC from fall 1996 until fal 2002. From 1996 to 2001, he worked part-time for the Department of Defense, codirecting the Optical Networking program at the DoD Laboratory for Telecommunications Sciences, Adelphi, MD, from 1999 to 2001. From 2001 to 2002, he was Chief Scientist at PhotonEx Corporation. For the last 15 years, his primary research area has been theoretical and computational studies of fiber optic communications. He has authored or coauthored more than 160 archival journal publications as well as numerous other publications and presentations. He has also edited two books. The equations and algorithms that he and his research group at UMBC have developed to model optical fiber transmission systems are used extensively in the telecommunications industry.

Dr. Menyuk is a Member of the Society for Industrial and Applied Mathematics and the American Physical Society. He is a Fellow of the Optical Society of America (OSA) and is a former UMBC Presidential Research Professor.

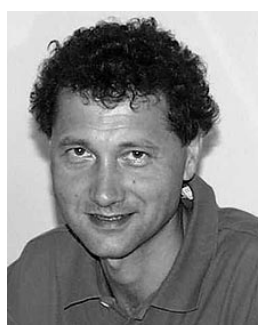

Andrea Galtarossa (M'88) received the degree in electronic engineering from the University of Padova, Padova, Italy, in 1984

In 1986, he received a Postgraduate Fellowship from Telettra Spa, Vimercate (IT), Italy, for research in wavelength-division-multiplexing (WDM) components. In 1987, he joined Saifo srl, Padova, Italy, a private company involved in measurements and teaching in optical systems. In 1990, he became an Assistant Professor in Electromagnetic Fields in the Department of Information Engineering, University of Padova. In 1998, he became an Associate Professor in Microwave Technology at the same university. He is the author or coauthor of more than 80 papers, and his current research activity is mainly in birefringent fibers and polarization-mode dispersion (PMD) measurements and modeling. 